\title{
ON THE CONSTRUCTION OF THE KUROSH LOWER RADICAL CLASS FOR ASSOCIATIVE RINGS
}

\author{
DARYL KREILING and TERRY L. JENKINS
}

(Received 18 november 1969)

Communicated by B. Mond

All rings considered are to be associative. For definitions not included in this paper see [2].

Let $R$ be a ring and $S$ a subring of $R$. We say that $S$ is accessible through zero extensions if there exists

$$
S=A_{0} \triangleleft A_{1} \triangleleft \cdots \triangleleft A_{n}=R
$$

such that $\left(A_{i} / A_{i-1}\right)^{2}=(0), i=1,2, \cdots n$.

We require the following lemma due to $\mathrm{S}$. E. Dickson.

LEMMA. [1, p. 447]. Let $R_{1}$ be a homomorphically closed class of rings containing the zero rings and having the additional property that if $I \in R_{1}$ is an ideal of $A$ with $(A / I)^{2}=(0)$, it follows that $A \in R_{1}$ (i.e., $R_{1}$ is closed under extensions by zero rings), then $R_{2}=\{A \mid$ each non-zero homomorphic image of $A$ contains a nonzero idea from $\left.R_{1}\right\}$ is the lower radical class containing $R_{1}$.

Let $P$ be a non-empty, homomorphically closed class of rings. Define the class $P_{1}$ by,

$P_{1}=\{A \mid A$ has a subring $S$ accessible through zero extensions, where $S \in P\}$.

THEOREM 1. With $P_{1}$ as defined above $P_{1}$ is homomorphically closed, contains the class of all zero rings and is closed under extensions by zero rings.

Proof. Let $A \in P_{1}$ and let $\phi$ be any homomorphism of $A$. Then there exists

$$
A_{0} \triangleleft A_{1} \triangleleft \cdots \triangleleft A_{n}=A
$$

where $A_{0} \in P$ and $\left(A_{i} / A_{i-1}\right)^{2}=(0)$. Hence,

$$
A_{0} \phi \triangleleft A_{1} \phi \triangleleft \cdots \triangleleft A_{n} \phi=A \phi,
$$

$A_{0} \phi \in P$ and $\left(A_{i} \phi / A_{i-1} \phi\right)^{2}=(0)$ so $A \phi \in P_{1}$ and $P_{1}$ is homomorphically closed.

If $A$ is a zero ring, $(0) \triangleleft A,(0) \in P$ and $(A /(0))^{2}=(0)$ so $A \in P_{1}$.

Finally, let $A$ be a ring such that there exists $I \triangleleft A, I \in P_{1}$ and $(A / I)^{2}=(0)$. Since $I \in P_{1}$ there exists 


$$
I_{0} \triangleleft I_{1} \triangleleft \cdots \triangleleft I_{n}=I \triangleleft A,
$$

$I_{0} \in P,\left(I_{i} / I_{i-1}\right)^{2}=(0), i=1,2, \cdots n$ and $(A / I)^{2}=(0)$. Hence, $A \in P_{1}$.

Let $L(P)$ denote the Kurosh lower radical class determined by $P$. Define

$P_{2}=\{A \mid$ each nonzero homomorphic image of $A$ contains a nonzero ideal from $\left.P_{1} \cdot\right\}$

Corollary 1. $P_{2}$ is a radical class containing $P$ and $L(P) \subseteq P_{2}$.

Proof. That $P_{2}$ is a radical class follows from the Lemma and Theorem 1. Since $L(P)$ is the smallest radical class containing $P, L(P) \subseteq P_{2}$.

COROLlary 2. If $L(P)$ (in particular if $P$ ) contains the class of zero rings, then $P_{2}=L(P)$.

Proof. Since $L(P)$ contains all zero rings and is closed under extensions $(I \in L(P), R / I \in L(P)$ implies $R \in L(P))$ [4, p. 13], we have that $P_{1} \subseteq L(P)$. Since $L(P)$ is a radical class $P_{2} \subseteq L(P)$. Hence, $P_{2}=L(P)$.

Remark. It is not true, in general, that $P_{2}=L(P)$. For example, let $P$ be the class of nontrivial simple rings plus the zero ring. Then $L(P)$ contains no simple zero rings but $P_{2}$ contains all zero rings. Hence in this case $L(P) \neq P_{2}$.

THEOREM 2. If the class $P$ is hereditary then $P_{2}$ is hereditary.

Proof. Let $A \in P_{1}$ and let $I$ be an ideal of $A$. Since $A \in P_{1}$ there exists

$$
A_{0} \triangleleft A_{1} \triangleleft \cdots \triangleleft A_{n}=A,
$$

where $A_{0} \in P$ and $\left(A_{i} / A_{i-1}\right)^{2}=(0)$. Now

$$
\left(A_{0} \cap I\right) \triangleleft\left(A_{1} \cap I\right) \triangleleft \cdots \triangleleft\left(A_{n} \cap I\right)=I .
$$

Since $P$ is hereditary and since $\left(A_{0} \cap I\right) \triangleleft A_{0}, A_{0} \cap I \in P$.

Moreover,

$$
\left(\left(A_{i} \cap I\right) /\left(A_{i-1} \cap I\right)\right)^{2}=(0)
$$

so $I \in P_{1}$. Thus $P_{1}$ is hereditary. Leavitt [3, p. 29] has shown that if $P_{1}$ is hereditary, then $P_{2}$ is hereditary.

REMARK. It is known that a radical class of associative rings contains all zero rings if and only if it contains all nilpotent rings [4, p. 18]. Since the lower Baer radical class, $B$, is the smallest radical class containing the nilpotent rings [2, p. 59], we have that $B \subseteq P_{2}$.

THEOREM 3. If $P$ contains no complete matrix rings over division rings then $P_{2}$ contains none. Hence, if $A$ is a ring with descending chain condition (d.c.c.) on left ideals and $W(A)$ denotes the classical Wedderburn radical of $A$, then $W(A)=$ $P_{2}(A)$. 
Proof. If $P$ contains no complete matrix ring over a division ring, then from the definition of $P_{1}$ it is clear that $P_{1}$ contains none. From the definition of $P_{2}$ it is then clear that $P_{2}$ contains no complete matrix rings over division rings. It then follows from [4, p. 19] that if $A$ is a ring with d.c.c. on left ideals, then $W(A)=$ $P_{2}(A)$.

\section{References}

[1] S. E. Dickson, 'A note on hypernilpotent radical properties for associative rings', Canadian Journal of Mathematics 19 (1967), 447-448.

[2] N. J. Divinsky, Rings and Radicals (University of Toronto Press, Toronto, 1965).

[3] Anthony E. Hoffman, The Constructions of the General Theory of Radicals (Doctoral Dissertation, University of Nebraska, 1966).

[4] Terry L. Jenkins, The Theory of Radicals and Radical Rings (Doctoral Dissertation, University of Nebraska, 1965).

Western Illinois University

University of Wyoming 